\title{
Crystal rotation in Cu single crystal micropillars: In situ Laue and electron backscatter diffraction
}

\author{
R. Maaß, ${ }_{2}^{1}$ S. Van Petegem, ${ }^{1}$ D. Grolimund, ${ }^{1}$ H. Van Swygenhoven, ${ }^{1, a)}$ D. Kiener, ${ }^{2}$ and \\ G. Dehm ${ }^{2}$ \\ ${ }^{1}$ Paul Scherrer Institute, Villigen CH-5232, Switzerland \\ ${ }^{2}$ Department of Materials Physics, Montanuniversität Leoben and Erich Schmid Institute of Materials \\ Science, Austrian Academy of Sciences, Leoben A-8700, Austria
}

(Received 1 January 2008; accepted 1 February 2008; published online 21 February 2008)

\begin{abstract}
In situ microdiffraction experiments were conducted on focused ion beam machined single crystal $\mathrm{Cu}$ pillars oriented for double slip. During deformation, the crystal undergoes lattice rotation on both the primary and critical slip system. In spite of the initial homogeneous microstructure of the $\mathrm{Cu}$ pillar, rotation sets in already at yield and is more important at the top of the pillar than at the bottom, demonstrating the inhomogeneous stress state during a microcompression experiment. The rotation results are confirmed by electron backscatter diffraction measurements. () 2008 American Institute of Physics. [DOI: 10.1063/1.2884688]
\end{abstract}

The origin of enhanced strengthening in single crystals when sample sizes are reduced to the micron scale is under heavy debate. ${ }^{1-3}$ The research progresses slowly because of the lack of appropriate nondestructive analysing techniques to investigate the initial and evolving microstructure of the sample. In situ Laue diffraction during microcompression of single crystal pillars is an excellent technique to reveal microstructural details in the pillar and to relate them to the observed deformation properties. ${ }^{4-6}$ For instance, it has been shown that a $2 \mu \mathrm{m}$ Au pillar can be stronger than a $10 \mu \mathrm{m}$ $\mathrm{Au}$ pillar, because the smaller pillar starts deforming on a slip system that is geometrically not predicted but selected because of the character of the preexisting strain gradient. ${ }^{5}$ In another case, a $10 \mu \mathrm{m} \mathrm{Ni}$ pillar was found to be extremely strong, a Laue scan revealed, however, the presence of a preexisting low angle grain boundary prior to loading. ${ }^{6}$ Plasticity could only be evidenced in the upper part of the pillar, demonstrating the barrier effect of the grain boundary. Here, we demonstrate for a $6 \mu \mathrm{m} \mathrm{Cu}$ pillar that, even in the absence of an initial strain gradient, the pillar deforms under an inhomogeneous stress state and crystal rotation is observed already at yield.

An in situ white beam Laue diffraction experiment is performed during microcompression of a single crystal $\mathrm{Cu}$ pillar, cut out by focused ion beam (FIB) milling from a bulk single crystal oriented for double slip as described elsewhere. ${ }^{7}$ The details of the Laue setup at the microdiffraction beamline (MicroXAS) of the Swiss Light Source (SLS) are given in [Ref. 5]. The $\mathrm{Cu}$ pillar was square with an initial dimension of $6 \times 6 \times 12 \mu \mathrm{m}^{3}$ and compressed with a constant loading rate of $1.5 \mu \mathrm{N} / \mathrm{s}$ up to $21 \%$ strain. The in situ Laue patterns were taken with a microfocused x-ray beam having a full width at half maximum (FWHM) of $2 \mu \mathrm{m}$ in the focal plane and an energy range from 2 to $22 \mathrm{keV}$. During compression, the beam was placed $5 \mu \mathrm{m}$ below the pillar top surface and a Laue pattern was recorded every $20 \mathrm{~s}$. Prior to loading and after deformation, the sample was scanned vertically and horizontally in search for microstructural inhomogeneities with a scanning step of $1 \mu \mathrm{m}$ and therefore

\footnotetext{
a)Electronic mail: helena.vs@psi.ch.
}

overlapping analyzed volumes. The deformed sample was also investigated by electron backscatter diffraction (EBSD) with a step size of $100 \mathrm{~nm}$ using a field emission scanning electron microscope (SEM) (Zeiss 1525) to compare the local orientation measurements with the findings of the Laue diffraction. The Laue scans prior to loading demonstrate a homogenous structure across the entire pillar, as is shown in Fig. 1(a) for the (3-11) reflection. All Laue reflections are nearly perfect symmetric, evidencing the absence of an excess dislocation density on a particular slip system, different to what was observed earlier in other pillars. ${ }^{4-6}$ Figure 1(d)

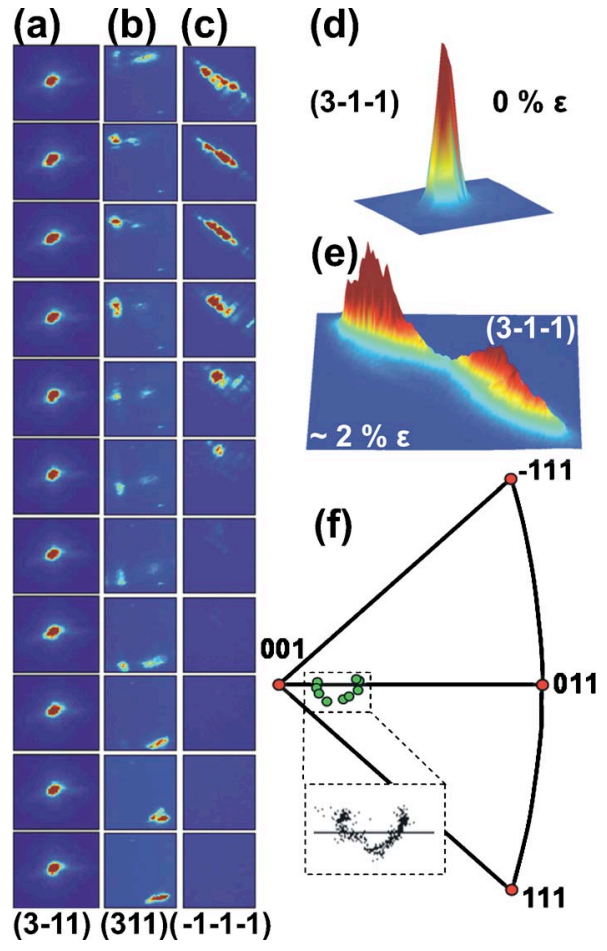

FIG. 1. (Color online) Laue spot scans along the vertical pillar axis demonstrating (a) the initial (3-11) peak and [(b)-(c)] the (311) and the (-1-1-1) reflection after deformation. (d) the (3-1-1) peak prior to loading and (e) splitting at $2 \%$ strain. (f) Compares the orientation distribution along a vertical scan line after $21 \%$ deformation obtained by XRD (green large points) and EBSD (inset with small black points). 


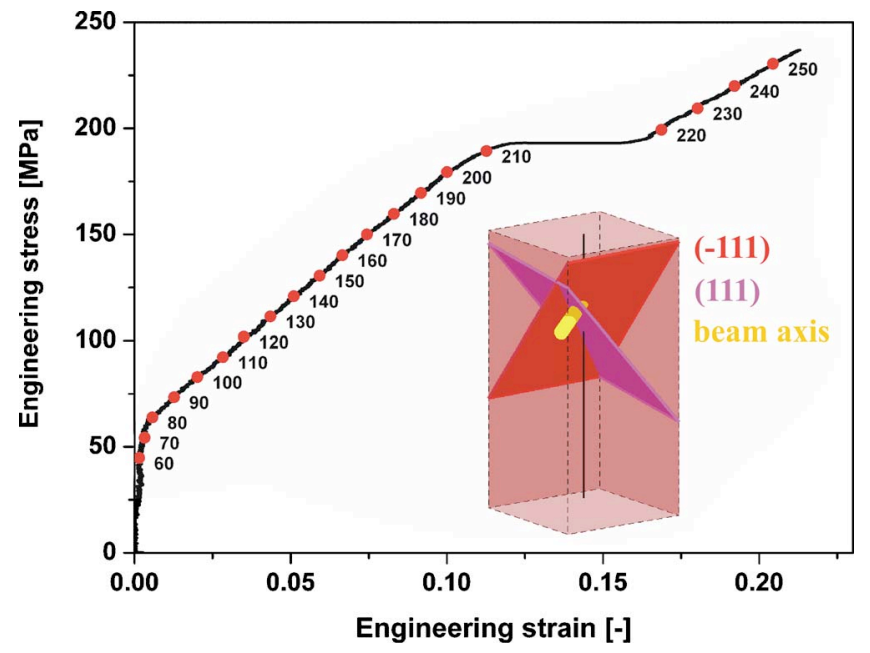

FIG. 2. (Color online) The stress-strain curve with red numbered dots corresponding to the Laue pattern number. Schematically the primary (111) and critical $(-111)$ slip planes are shown relative to the incoming beam axis during the in situ experiment.

shows the Lorentzian shape of the (3-1-1) reflection prior to loading in 3D, which evidences the same FWHM (minor/ major axis $=3.0 / 1.8$ pixels, obtained from a two dimensional Pearson VII fit) as a Si (422) reference peak located close to the $\mathrm{Cu}$ peak. The stress-strain curve recorded during the in situ experiment is shown in Fig. 2. Red data points correspond to the strains where every tenth Laue pattern was taken. Linear elastic loading is observed till $\sim 62 \mathrm{MPa}$, where macroscopic yielding occurs, followed by a linear strain hardening up to $12 \%$ strain. Here, a large strain burst of $\sim 4 \%$ strain is observed after which the sample hardens with a slightly lower hardening rate. The observed yield strength lies within the scatter of previous in situ compression tests performed in a SEM for similar sample sizes using a different indenter, ${ }^{8}$ and is higher compared to the bulk value of single crystal copper, which yields at $\sim 1-2 \mathrm{MPa}$ for single slip and $\sim 3 \mathrm{MPa}$ for double slip. ${ }^{9,10}$

From the initial Laue diffraction pattern, the crystallographic direction of the compression axis is with [0.02 0.12 $0.99] 7^{\circ}$ off from the [001] direction, which means that the predicted primary (111) and critical slip planes $(-111)$ both have a Schmid factor (SF) equal to 0.45 . The orientations of these planes are shown schematically in the inset of Fig. 2. The yellow line represents the incoming beam axis. As two slip systems have equal values for the SF, stage II type work hardening for multiple glide in single crystals is to be expected. The measured strain hardening rates $d \sigma / d \varepsilon$ $=1.2 \mathrm{GPa}$ (before burst) and $0.9 \mathrm{GPa}$ (after burst) are comparable to those measured for bulk $\sim\langle 001\rangle$ oriented copper. ${ }^{11}$ The decrease of $d \sigma / d \varepsilon$ after the burst points toward the presence of a mechanism that partially relieved the internal network responsible for the hardening. ${ }^{12}$

For each recorded Laue pattern during deformation, the actual vertical crystal axis of the sample can be traced in the stereographic 001-projection. During deformation, the vertical crystal axis shifts toward the [011] pole, following the connecting line between the [001] and [011] pole. Comparison of the initial and the final ([ $\left.\left[\begin{array}{lll}0.03 & 0.24 & 0.97\end{array}\right]\right)$ orientations of the vertical crystal axis demonstrates a $7^{\circ}$ rotation occurring within the probed volume during the $21 \%$ strain. Assuming that the total rotation is due to slip on the predicted

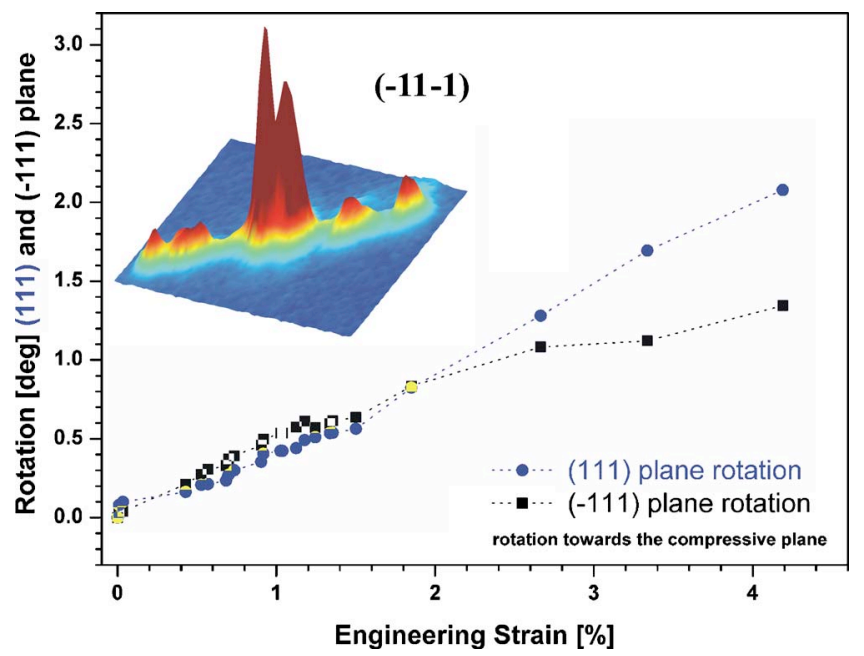

FIG. 3. (Color online) Successive rotations of the primary (111) and critical $(-111)$ slip plane resolved as a function of strain. The inset shows the $(-11-1)$ reflection split into six subreflections just before the strain burst in Fig. 2.

(-111) and (111) planes, it is possible to calculate the amount of rotation for each slip system. Figure 3 shows that roughly equal amounts of rotation are observed up to $2 \%$ strain and that rotation starts already at yield. Above $2 \%$ strain, the change in rotation with respect to strain $d \theta / d \varepsilon$ decreases for the $(-111)$ plane. The sudden difference in $d \theta / d \varepsilon$ for the two slip planes is reflected in the (3-1-1) diffraction peak. The initial homogenously distributed intensity splits in the rotation direction of the $(-111)$ plane at $2 \%$ strain [Fig. 1(e)], indicating the formation of a small angle grain boundary/polygonization in the probed volume with its plane intersecting the glide plane, hindering continued uniform rotation toward the compressive plane.

As deformation proceeds, collective peak movement is accompanied by large streaking and all detected peaks split further to form multiple subreflections. This reflects low angle boundary formation, where geometrically necessary dislocations (GNDs) arrange to accommodate lattice curvature. Just before the observed strain burst one can observe six individual subpeaks of the $(-11-1)$ reflection along the splitting direction for the $(-111)$ slip plane, as is shown in the inset of Fig. 3. The total misorientation represented in this peak splitting is $1.23^{\circ}$. Assuming that the misorientation is due to GNDs forming a boundary, one can relate the misorientation angle $\Theta$ to $b / h=2 \sin \Theta / 2, b$ being the Burger vector here taken to be $0.2556 \mathrm{~nm}$ and $h$ the distance between GNDs in the formed geometrically necessary boundary (GNB). Also, assuming that the GNB extends over the probed area $(D=2 \mu \mathrm{m})$, the dislocation density can be estimated by $n^{+}=1 / D h,{ }^{13}$ resulting in an estimated GND density $\rho_{\mathrm{GND}}=4 \times 10^{13} \mathrm{~m}^{-2}$. In view of the partial pillar volume illuminated during the in situ test, it can therefore be concluded that the sample must already contain numerous low angle boundaries just before the strain burst. By investigating the $(-11-1)$ reflection as a function of time, it can also be seen that the boundary formation before and after the burst is compatible with the $(-111)$ slip plane, but due to the close proximity of the streaking direction, its slip system cannot be assigned unambiguously. The observed burst however corresponds to a peak shift, which follows the rotation direction of the (111) slip plane. 


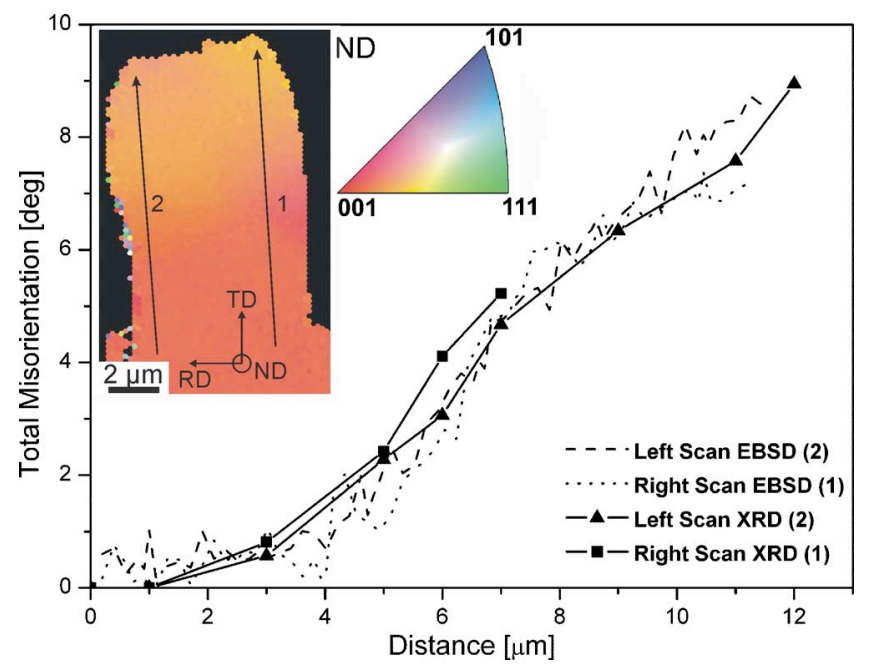

FIG. 4. (Color online) Two vertical EBSD scans compared with the corresponding X-ray scan data. All curves display the total misorientation relative to the base orientation of the pillar plotted as a function of distance from the pillar base. The color code of the EBSD scan reflects the normal crystal direction as in the shown standard triangle.

After deformation, three vertical scans along the sample were performed, one along the central pillar axis and the others $\pm 3 \mu \mathrm{m}$ off the center. A vertical scan along the left axis is shown in Fig. 1(b) for the (311) and in Fig. 1(c) for the (-1-1-1) peak. From the peak shift and change in intensity spread along the vertical scanning direction, it is clear that rotation occurred predominantly in the upper part of the sample, as is demonstrated by the appearance of new reflections such as the intensity distribution of the (311) peak in the upper part of the pillar and the nearly unchanged shape of the reflections in the bottom of the pillar. These observations show that the stress state is not uniformly distributed over the sample even in the absence of a taper, as has been found in Ni micropillars. ${ }^{6}$

The rotations captured in Figs. 1(b), 1(c), and 3 have been directly compared with conventional electron backscattering diffraction technique (EBSD). EBSD measurements were performed on a (100) plane that was cut out of the pillar (using FIB) along its [001] axis. Figure 1(f) demonstrates a comparison of the change in vertical crystal axis obtained by Laue pattern analysis from the postdeformation scan and the comparative EBSD measurements for the same scan. It is, however, important to keep in mind that the x-ray measurements are done by probing the entire sample in transmission, whereas EBSD reveals information from a several tens of nanometer thick layers of the center region uncovered by FIB machining after deformation, but, therefore, with high lateral resolution. The misorientations determined from the EBSD data are measured relative to the undeformed bottom of the pillar. The results are shown in Fig. 4 together with the rotations derived from the Laue scans along a similar vertical axis.

The inset in Fig. 4 shows the cross section on which the EBSD measurements were performed. The asymmetric pillar shape is not a result of bending during deformation, but the shape modification occurred unintentionally during a plasma cleaning procedure prior to SEM-EBSD studies to remove carbon contamination. The step size $(100 \mathrm{~nm})$ and beam diameter $(<10 \mathrm{~nm})$ during EBSD measurements are finer than those of the Laue measurements $(1 \mu \mathrm{m})$. On the other hand, the nominal orientation resolution of the EBSD system is with $0.5^{\circ}$ worse compared to the resolution of the Laue pattern's $0.04^{\circ}$, which is predominantly determined by the charge-coupled device-pixel size and the geometry. Figure 4 shows close agreement between the amount of rotation derived during a Laue scan over the pillar and the amount of misorientation derived from EBSD scans. The two techniques agree in the direction and amount of misorientation along the scan lines 1 and 2, as is demonstrated by the paths in the stereographic plots in Fig. 1(f).

In summary, in situ Laue microcompression was used to reveal the successive crystal rotation on both the primary and critical slip plane in a single crystal $\mathrm{Cu}$ micropillar initially oriented for double slip. Resolved as a function of strain, the data demonstrate a classical stage II type work hardening in spite of the higher yield stress. In situ Laue shows that rotation already starts upon yielding and occurs nonhomogenously along the pillar's vertical axis, demonstrating the asymmetric stress states of a micropillar compression test. The latter has been confirmed by EBSD measurements performed on a cross section of the same pillar after deformation. Despite the higher yield strength, the presented results do agree with classical plasticity and therefore do not support the picture of dislocation starvation at this length scale. ${ }^{14,15}$ The cause for the increased strength remains to be explored.

The authors thank S. Brandstetter and L. Thilly for help during beam time and P. M. Derlet for useful discussions. H.V.S. thanks the Swiss National Science Foundation and the European Commission (6th Framework) for financial support of the project NANOMESO. The authors also thank $\mathrm{C}$. Borca and M. Willimann from the MicroXAS/SLS and Hysitron, Inc., for technical support.

${ }^{1}$ M. D. Uchic, D. M. Dimiduk, J. N. Florando, and W. D. Nix, Science 305, 986 (2004).

${ }^{2}$ J. R. Greer, W. C. Oliver, and W. D. Nix, Acta Mater. 53, 1821 (2005).

${ }^{3}$ C. A. Volkert and E. T. Lilleodden, Philos. Mag. 86, 5567 (2006).

${ }^{4}$ R. Maaß, D. Grolimund, S. Van Petegem, M. Willimann, M. Jensen, H. Van Swygenhoven, T. Lehnert, M. A. M. Gijs, C. A. Volkert, E. T. Lilleodden, and R. Schwaiger, Appl. Phys. Lett. 89, 151905 (2006).

${ }^{5}$ R. Maaß, S. Van Petegem, H. Van Swygenhoven, P. M. Derlet, C. A. Volkert, and D. Grolimund, Phys. Rev. Lett. 99, 145505 (2007).

${ }^{6}$ R. Maaß, S. Van Petegem, D. Grolimund, H. Van Swygenhoven, and M. D. Uchic, Appl. Phys. Lett. 91, 131909 (2007).

${ }^{7}$ D. Kiener, M. Rester, S. Scheriau, B. Yang, R. Pippan, and G. Dehm, Int. J. Mat. Res. 98, 1047 (2007).

${ }^{8}$ D. Kiener, C. Motz, T. Schöberl, M. Jenko, and G. Dehm, Adv. Eng. Mater. 8, 1119 (2006).

${ }^{9}$ J. Diehl, Z. Metallkd. 47, 411 (1956).

${ }^{10}$ P. Franciosi and A. Zaoui, Acta Metall. 30, 2141 (1982).

${ }^{11}$ T. Takebuchi, J. Phys. Soc. Jpn. 40, 741 (1976).

${ }^{12}$ U. F. Kocks, Acta Metall. 8, 345 (1960).

${ }^{13}$ R. I. Barabash, G. E. Ice, and F. J. Walker, J. Appl. Phys. 93, 1457 (2003).

${ }^{14}$ A. S. Budiman, S. M. Han, J. R. Greer, N. Tamura, J. R. Patel, and W. D. Nix, Acta Mater. 56, 602 (2007).

${ }^{15}$ J. R. Greer and W. D. Nix, Phys. Rev. B 73, 245410 (2006). 